CARTA AL EDITOR

\title{
DISCREPANCIAS SOBRE LA VERSIÓN CEFÁLICA EXTERNA EN LAS GUÎAS DE PRÁCTICA CLÍNICA PARA EL MANEJO DE PARTO PODÁLICO
}

\section{DISCREPANCIES ABOUT EXTERNAL CEPHALIC VERSION IN CLINICAL PRACTICE GUIDELINES FOR THE MANAGEMENT OF BREECH DELIVERY}

\author{
Daniel Fernandez-Guzman (1),2,a, \\ Ramón Ruesta-Berdejo (13,3,b, Jose Galvez-Olortegui(1) 1,4,c, \\ Juan Carlos Torrejon-Becerra ${ }^{1,5, \mathrm{~d}}$ \\ 1 Unidad Generadora de Evidencias y Vigilancia Epidemiológica. Scientia \\ Clinical and Epidemiological Research Institute. Trujillo, Perú. \\ 2 Escuela Profesional de Medicina Humana, Universidad Nacional de San \\ Antonio Abad del Cusco. Cusco, Perú. \\ 3 Escuela Profesional de Medicina Humana, Universidad Nacional de Piura, \\ Piura, Perú. \\ 4 Servicio de Oftalmología, Hospital Universitario Central de Asturias, \\ Oviedo, España. \\ 5 Servicio de Ginecología y Obstetricia, Hospital Universitario Central de \\ Asturias, Oviedo, España. \\ a Bachiller de Medicina; ${ }^{b}$ estudiante de Medicina, ${ }^{\mathrm{c}}$ médico cirujano, magíster \\ en Investigación Clínica; ${ }^{\circledR}$ Médico Especialista en Ginecología y Obstetricia.
}

Sr. Editor: La versión cefálica externa (VCE) es un procedimiento manual que, a través de la rotación externa, busca conseguir una posición cefálica en fetos con presentación de nalgas, aumentando así la posibilidad de un parto por vía vaginal ${ }^{(1)}$. Sin embargo, la calidad metodológica y las recomendaciones de las guías de práctica clínica (GPC) sobre el manejo del parto continúan siendo cuestionables. Por lo que, el objetivo del presente artículo fue revisar las discrepancias entre las GPC internacionales de referencia y la peruana en el manejo de la presentación de nalgas, enfocándonos en las recomendaciones relacionadas con la VCE.

Se realizó una búsqueda en Pubmed/Medline con los términos: "external cephalic version" y "guidelines", "recommendations" o "clinical practice guidelines" durante el periodo 2007-2021, en idioma inglés o español, recuperándose solo la GPC americana. Se revisaron las páginas web oficiales de las instituciones de desarrollo de guías nacionales y de las sociedades de ginecología-obstetricia del Reino Unido, Estados Unidos y España, por ser países desarrollados, tener una actualización permanente de sus GPC y por los años de implementación de estas prácticas en sus hospitales. Se

Citar como: Fernández-Guzmán D, Ruesta-Berdejo R, Galvez-Olortegui J, Torrejon-Becerra JC. Discrepancias sobre la versión cefálica externa en las guías de práctica clínica para el manejo de parto podálico. Rev Peru Med Exp Salud Publica. 2021;38(3):476-8. doi: https://doi.org/10.17843/rpmesp.2021.383.7304.

Correspondencia: Juan Carlos Torrejon-Becerra, juancarlostorrejon@gmail.com

Recibido: 08/02/2021 Aprobado: 23/06/2021 En línea: 12/08/2021 comparó con la última actualización de la GPC peruana, desarrollada por el Instituto Nacional Materno Perinatal y el Ministerio de Salud, como representante de un país en vías de desarrollo. Las recomendaciones encontradas sobre el manejo del parto podálico fueron recopiladas y analizadas de forma independiente por dos de los autores (DFG y RRB), revisadas por un tercer autor (JCTB) y se resolvieron las diferencias por consenso.

Las GPC de los países desarrollados recomiendan usar la ultrasonografía para confirmar el diagnóstico de presentación de nalgas, ofrecer y realizar como primera medida la VCE; si las gestantes no cumplen los criterios o ante la negativa para que le realicen el procedimiento, tiene el derecho de elegir entre un intento de parto vaginal (IPV) o cesárea. Por otro lado, la GPC peruana recomienda terminar la gestación mediante cesárea programada; solo se indica parto vaginal cuando la gestante se encuentra en periodo expulsivo (Tabla 1).

La GPC peruana favorece la realización de un número de cesáreas mayor a las necesarias. Se conoce bien el aumento de la morbimortalidad materna durante la cesárea, comparada con el parto vaginal, así como sus repercusiones en futuros embarazos. Adicionalmente, la cesárea previa constituye la indicación aislada más frecuente para un nuevo parto por cesárea. Esto condicionará un desafío tanto para la paciente como para el personal sanitario, ya que una segunda cesárea se asocia con un mayor riesgo de complicaciones quirúrgicas, y placentación anormal en embarazos posteriores. Por otro lado, el IPV después de una cesárea se puede asociar con un mayor riesgo de complicaciones maternas e infantiles, y una proporción sustancial de mujeres que intentan un parto vaginal después de una cesárea pueden requerir una cesárea de emergencia.

A pesar de que la cesárea reduce la morbimortalidad perinatal a corto plazo en comparación al parto vaginal (2), existen estudios que están evaluando las repercusiones a largo y mediano plazo. El IPV en la presentación de nalgas es una opción segura cuando el personal está capacitado y se cuenta con criterios estrictos de selección ${ }^{(3)}$. Asimismo, la VCE es una opción razonable; su implementación consigue disminuir la frecuencia de cesáreas y aumenta la posibilidad del parto vaginal ${ }^{(4)}$; es un procedimiento seguro ${ }^{(5)}$, con resultados similares entre mujeres con y sin antecedente de cesárea ${ }^{(6)}$. Esta estrategia debería fomentarse en países de Latinoamérica, como Perú, considerando sus claros beneficios y complicaciones mínimas (cambios transitorios de la frecuencia cardiaca fetal [FCF] [5,7\%], la hemorragia feto-materna [3,7\%], alteración persistente de la FCF $[0,4 \%]$, sangrado vaginal $[0,4 \%]$, y rotura de placenta $[0,12 \%]){ }^{(7)}$. Además, podría realizarse en centros hospitalarios que cuenten con personal adecuadamente capacitado, lo que permitiría monitorear continuamente tanto a la madre como al feto antes, durante y después del procedimiento, y realizar una cesárea si se presentase la necesidad ${ }^{(3)}$. Dado que la curva de 
Tabla 1. Comparación de las recomendaciones sobre el manejo de la presentación de nalgas

\begin{tabular}{|c|c|c|c|c|}
\hline Recomendaciones & Guía británica & Guía americana & Guía española & Guía peruana \\
\hline Año de actualización & 2017 & $2018 / 2020$ & $2011 / 2014$ & 2007 \\
\hline $\begin{array}{l}\text { Ultrasonido para confirmar la mala } \\
\text { presentación del feto }\end{array}$ & Recomendado & Recomendado & Recomendado & Recomendado \\
\hline VCE & Recomendado & Recomendado & Recomendado & No se indica \\
\hline $\begin{array}{l}\text { Informar a las mujeres sobre los benefi- } \\
\text { cios y riesgos de la VCE }\end{array}$ & Recomendado & Recomendado & Recomendado & No se indica \\
\hline Contraindicaciones de la VCE & $\begin{array}{l}\text { No hay consenso general sobre la } \\
\text { elegibilidad de las contraindicaciones }\end{array}$ & $\begin{array}{l}\text { Casos en los que el parto } \\
\text { vaginal no es clínicamente } \\
\text { apropiado }\end{array}$ & $\begin{array}{l}\text { Existen contraindicaciones absolu- } \\
\text { tas y relativas }\end{array}$ & No se indica \\
\hline VCE en gestantes con cesárea previa & $\begin{array}{l}\text { No parece aumentar el riesgo ni } \\
\text { disminuir la tasa de éxito }\end{array}$ & $\begin{array}{l}\text { Mismo grado de éxito que las } \\
\text { gestantes sin cesárea previa }\end{array}$ & $\begin{array}{l}\text { Procedimiento seguro y tasas } \\
\text { de éxito de la VCE similar entre } \\
\text { aquellas gestantes sin antecedentes } \\
\text { de cesárea }\end{array}$ & No se indica \\
\hline VCE durante fase activa del parto & No se indica & No se indica & $\begin{array}{l}\text { Factible, con probabilidad de éxito } \\
\text { si se utiliza un tocolítico y si se } \\
\text { trata de una paciente multípara }\end{array}$ & \\
\hline $\begin{array}{l}\text { VCE por un médico capacitado en una } \\
\text { instalación adecuada }\end{array}$ & Recomendado & Recomendado & Recomendado & No se indica \\
\hline $\begin{array}{l}\text { Cuándo se debe realizar el intento de } \\
\text { VCE }\end{array}$ & $\begin{array}{c}\text { Se puede ofrecer a partir de las } 36 \\
\text { semanas de gestación. En mujeres } \\
\text { multíparas se prefiere a partir de la } \\
\text { semana } 37\end{array}$ & $\begin{array}{l}\text { Desde las } 36 \text { semanas de } \\
\text { gestación }\end{array}$ & $\begin{array}{l}\text { A partir de las } 37 \text { semanas de } \\
\text { gestación }\end{array}$ & No se indica \\
\hline Monitorización fetal durante la VCE & $\begin{array}{l}\text { Monitorización fetal previa, ecografía } \\
\text { durante el procedimiento y evaluación } \\
\text { fetal posterior a la VCE }\end{array}$ & $\begin{array}{l}\text { Monitorización fetal o perfil } \\
\text { biofísico antes de la VCE, } \\
\text { ecografía durante el proce- } \\
\text { dimiento y evaluación fetal } \\
\text { después de la VCE }\end{array}$ & $\begin{array}{l}\text { Monitorización fetal previa, eco- } \\
\text { grafía durante el procedimiento y } \\
\text { evaluación fetal posterior a la VCE }\end{array}$ & No se indica \\
\hline $\begin{array}{l}\text { Tocolíticos durante } \\
\text { la VCE }\end{array}$ & Se recomiendan los betamiméticos & Recomendado & $\begin{array}{l}\text { Se recomienda Ritodrine o Ato- } \\
\text { siban }\end{array}$ & No se indica \\
\hline $\begin{array}{l}\text { Analgesia durante } \\
\text { la VCE }\end{array}$ & No se recomienda de rutina & $\begin{array}{l}\text { De recomendar alguna se } \\
\text { recomienda la analgesia } \\
\text { neuroaxial }\end{array}$ & $\begin{array}{l}\text { No hay un consenso de cuál es la } \\
\text { mejor estrategia analgésica para } \\
\text { la VCE }\end{array}$ & No se indica \\
\hline $\begin{array}{l}\text { Inmunoglobulina antiD para mujeres } \\
\text { Rh negativas en quienes se realice } \\
\text { la VCE }\end{array}$ & Recomendado & Recomendado & Recomendado & No se indica \\
\hline $\begin{array}{l}\text { Decisión de la paciente sobre el modo } \\
\text { de parto en presentación de nalgas }\end{array}$ & Recomendado & Recomendado & Recomendado & $\begin{array}{l}\text { No se reco- } \\
\text { mienda }\end{array}$ \\
\hline $\begin{array}{l}\text { Opción de parto vaginal en casos de } \\
\text { presentación de nalgas persistente }\end{array}$ & Si cumple criterios de IPV & Si cumple criterios de IPV & Si cumple criterios de IPV & $\begin{array}{l}\text { Aquellos con } \\
\text { periodo expulsi- } \\
\text { vo avanzado }\end{array}$ \\
\hline $\begin{array}{l}\text { Discutir opciones de la vía de culmina- } \\
\text { ción del embarazo con la gestante si la } \\
\text { presentación de nalgas se diagnostica } \\
\text { durante el trabajo de parto. }\end{array}$ & Recomendado & No se indica & No se indica & Recomendado \\
\hline $\begin{array}{l}\text { Disponibilidad de instalaciones para el } \\
\text { parto por cesárea }\end{array}$ & Recomendado & Recomendado & Recomendado & Recomendado \\
\hline $\begin{array}{l}\text { Inducción del trabajo de parto tras } \\
\text { VCE exitosa }\end{array}$ & No está indicada, salvo excepciones & $\begin{array}{l}\text { No hay evidencia que apoye } \\
\text { esta práctica de forma ru- } \\
\text { tinaria }\end{array}$ & $\begin{array}{l}\text { Pueden considerarse casos de } \\
\text { ausencia de progresión de la dila- } \\
\text { tación con hipodinamia primaria }\end{array}$ & No se indica \\
\hline
\end{tabular}

ACOG: Colegio Americano de Obstetras y Ginecólogos; RCOG: Real Colegio de Obstetras y Ginecólogos; SEGO: Sociedad Española de Ginecología y Obstetricia; GPC: Guía de Práctica Clínica; VCE: versión cefálica externa; IPV: intento de parto Vaginal

Guía británica: External Cephalic version and reducing the incidence of term breech presentation: Green-top Guideline No. 20a. BJOG 2017; 124(07):e178-e192 and Royal College of Obstetricians and Gynaecologists. Management of Breech Presentation: Green-top Guideline No. 20b. BJOG An Int J Obstet Gynaecol. 2017;124(7):e151-e177. doi:10.1111/14710528.14465

Guía americana: ACOG Committee Opinion No. 745: Mode of Term Singleton Breech Delivery. Obstet Gynecol. 2018;132(2):e60-e63. doi:10.1097/AOG.0000000000002755 and External Cephalic Version. ACOG Practice Bulletin No. 221. American College of Obstetricians and Gynecologists. Obstet Gynecol 2020;135:e203-12. Disponible en: https://www.acog. org/clinical/clinical-guidance/practice-bulletin/articles/2020/05/external-cephalic-version

Guía española: Sociedad Española de Ginecología y Obstetricia. Guía Práctica de asistencia: Versión Cefálica Externa. ProSEGO. España. 2014: 1-8. And Sociedad Española de Ginecología y Obstetricia. Guía Práctica de asistencia: Parto en la presentación de nalgas a término. ProSEGO. España. 2011: 1-23.

Guía peruana: Instituto Nacional Materno Perinatal. Guías de Práctica Clínica y de Procedimientos en Obstetricia y Perinatología. Edición N. ${ }^{\circ}$ 1. Lima, Perú: MINSA; $2007: 233-236$. 
aprendizaje para realizar la VCE es mucho más corta y sencilla comparada con otros procedimientos como la cesárea, se logran tasas de éxito importantes en nulíparas, incluso siendo un médico principiante ${ }^{(8)}$ y podría ser fácilmente aplicable en la formación de ginecoobstetras en Perú, especialmente a través de talleres con simuladores guiados por ginecólogos expertos.

En conclusión, las GPC para el manejo del parto podálico presentan discrepancias importantes. La GPC peruana se encuentra desactualizada, y no presenta valoración o recomendación alguna sobre la VCE en el manejo del parto podálico, pese al bajo riesgo de eventos adversos y la posibilidad de reducir significativamente la frecuencia de partos por cesárea. Se debe considerar la VCE en todos los casos de fetos con presentación de nalgas si no presentasen contraindicaciones,

\section{REFERENCIAS BIBLIOGRÁFICAS}

1. Committee on Practice Bulletins-Obstetrics (ACOG). External Cephalic Version. Obstetrics \& Gynecology. 2020; 135(5), 203-212. doi:10.1097/AOG.0000000000003837.

2. Hofmeyr GJ, Hannah M, Lawrie TA. Planned caesarean section for term breech delivery. Vol. 2015, Cochrane Database of Systematic Reviews. John Wiley and Sons Ltd; 2015. doi: 10.1002/14651858. cd006553.pub3.

3. Goffinet F, Carayol M, Foidart J-M, Alexander S, Uzan S, Subtil D, et al. Is planned vaginal delivery for breech presentation at term still an option? Results of an observational prospective survey in France and Belgium. Am J Obstet Gynecol. 2006;194(4):1002-11. doi: 10.1016/j. ajog.2005.10.817.

4. Rijnders M, Offerhaus P, Van Dommelen P, Wiegers T, Buitendijk S. Prevalence, outcome, and women's experiences of external cephalic version in a low-risk population. Birth. 2010;37(2):124-33. doi: 10.1111/j.1523-536x.2010.00392.x. respetando la decisión de las gestantes respecto a la vía de parto y considerando las condiciones adecuadas para poder realizarlo. Recomendamos la enseñanza de la VCE dentro de los programas de educación médica. Finalmente, la VCE debería ser implementada en países en vías de desarrollo, incluido Perú, a través de su GPC, como principal medida para reducir las cesáreas programadas por fetos con presentación de nalgas y evitar sus futuras consecuencias negativas.

Contribución: DFG concibió el artículo. RRB, JGO y JCTB redactaron y aprobaron el manuscrito.

Financiamiento: El presente estudio fue autofinanciado.

Conflictos de interés: Los autores no refieren tener algún conflicto de interés.
5. Son M, Roy A, Grobman WA, Miller ES. Association Between Attempted External Cephalic Version and Perinatal Morbidity and Mortality. Obstet Gynecol. 2018;132(2):365-70. doi: 10.1097/ aog.0000000000002699.

6. Homafar M, Gerard J, Turrentine M. Vaginal Delivery After External Cephalic Version in Patients With a Previous Cesarean Delivery: A Systematic Review and Meta-analysis. Obstet Gynecol. 2020;136(5):965-71. doi: 10.1097/aog.00000000000040657.

7. Collaris RJ, Oei SG. External cephalic version: A safe procedure? a systematic review of version-related risks. Acta Obstet Gynecol Scand. 2004;83(6):511-8. doi: 10.1111/j.0001-6349.2004.00347.x.

8. Kim SY, Han JY, Chang EH, Kwak DW, Ahn HK, Ryu HM, et al. Evaluation of the learning curve for external cephalic version using cumulative sum analysis. Obstet Gynecol Sci. 2017;60(4):343-9. doi: 10.5468/ogs.2017.60.4.343. 